\title{
Survival in patients with synchronous liver metastases in central and northern Denmark, 1998 to 2009
}

This article was published in the following Dove Press journal:

Clinical Epidemiology

19 July 2011

Number of times this article has been viewed

\author{
Morten Høyer ${ }^{1,2}$ \\ Rune Erichsen' \\ Per Gandrup ${ }^{3}$ \\ Mette Nørgaard' \\ Jacob Bonde Jacobsen' \\ 'Department of Clinical Epidemiology, \\ Aarhus University Hospital, Aarhus, \\ Denmark; ${ }^{2}$ Department of Oncology, \\ Aarhus University Hospital, \\ Aarhus, Denmark; ${ }^{3}$ Department of \\ Gastrointestinal Surgery, Aarhus \\ University Hospital, Aalborg, Denmark
}

Correspondence: Morten Høyer Department of Oncology, Aarhus University Hospital, Nørrebrogade 44, 8000 Aarhus C, Denmark

Tel +4589492529

Fax +4589492530

Email hoyer@aarhus.rm.dk
Objective: In Denmark, the strategy for treatment of cancer with metastases to the liver has changed dramatically during the period 1998 to 2009, when multidisciplinary care and a number of new treatments were introduced. We therefore examined the changes in survival in Danish patients with colorectal carcinoma (CRC) or other solid tumors (non-CRC) who had liver metastases at time of diagnosis.

Study design and methods: We included patients diagnosed with liver metastases synchronous with a primary cancer (ie, a solid cancer diagnosed at the same date or within 60 days after liver metastasis diagnosis) during the period 1998 to 2009 identified through the Danish National Registry of Patients. We followed those who survived for more than 60 days in a survival analysis $(n=1021)$. Survival and mortality rate ratio $(M R R)$ at 1,3 , and 5 years stratified by year of diagnosis were estimated using Cox proportional hazards regression analysis.

Results: In the total study population of 1021 patients, 541 patients had a primary CRC and 480 patients non-CRC. Overall, the 5-year survival improved from 3\% (95\% confidence interval [CI]: 1\%-6\%) in 1998-2000 to 10\% (95\% CI: 6\%-14\%) in 2007 to 2009 (predicted value). The 5-year survival for CRC-patients improved from 1\% (95\% CI: $0 \%-5 \%)$ to $11 \%(95 \% \mathrm{CI}$ : $6 \%-18 \%)$ whereas survival for non-CRC patients only increased from 5\% (95\% CI: $1 \%-10 \%)$ to $8 \%$ (95\% CI: $4 \%-14 \%)$.

Conclusion: We observed improved survival in patients with liver metastases in a time period characterized by introduction of a structured multidisciplinary care and improved treatment options. The survival gain was most prominent for CRC-patients.

Keywords: liver metastases, colorectal cancer, noncolorectal cancer, survival improvement

\section{Introduction}

The liver is a common site of metastases and gastrointestinal-, lung- and breast cancers frequently give rise to liver metastases. ${ }^{1}$ Liver metastasis is usually considered to be a manifestation of end stage disease. However, in patients with colorectal cancer (CRC) metastases, the disease often presents with oligometastases relatively early on and the liver is often the only site of metastasis. Hence, at the time of CRC-diagnosis, $15 \%$ of patients have metastases to the liver and in approximately $75 \%$ of these, the metastases are confined to the liver. ${ }^{2}$ In general, systemic therapy is the preferred treatment for most patients with liver metastases, but patients with a limited number and size of the metastases and with favorable histology, especially CRC, should be considered for focal therapy. ${ }^{3-7}$ A large number of systemic therapies for patients with metastatic cancer have been introduced during the last 10 years and over the same period surgery 
and nonsurgical techniques for focal ablation of metastases in the liver have improved considerably. ${ }^{8}$

The Danish National Board of Health launched The National Cancer Treatment Plans I and II in 2000 and 2005 aiming at improving cancer treatment in the country. ${ }^{9}$ The major issues were improvements in diagnostics, surgery, radiation therapy, and chemotherapy for cancer, and major government investments have followed the program. Assessing whether these efforts have resulted in real improvement in terms of survival outcomes is thus of great interest. We therefore conducted a cohort study in Northern Denmark to study time changes in survival in patients with liver metastases synchronously to CRC and non-CRC during 1998 to 2009. We chose only to evaluate patients with synchronous liver metastases because metachronous metastases are known to be incompletely registered. Due to differences in biology and therapy options, the study evaluated patients with CRC and non-CRC liver metastases separately.

\section{Material and methods}

The study was conducted in the Central and the North Denmark Regions (2 of 5 Danish regions), with a combined population of 1.8 million. The National Health Service provides tax-supported health care for all inhabitants of Denmark, guaranteeing free access to hospitals. A 10-digit civil registration number has been assigned to all residents by the Central Office of Civil Registration since 1968. ${ }^{10}$ This number, unique to each Danish resident, is used in all Danish registries, allowing unambiguous individual-level data linkage.

\section{Identification of liver metastases cancer patients}

Through the Danish National Registry of Patients (DNRP), we identified all patients who had a first time diagnosis of liver metastases in the period January 1, 1998 through December 31, 2009 and no previous cancer diagnosis recorded in the DNRP. DNRP contains information about all admissions from nonpsychiatric hospitals in Denmark since $1977 .{ }^{11}$ Outpatient and emergency room visits at hospitals have been included since 1995 . This registry includes information on civil registration number, dates of admission and discharge, surgical procedure(s) performed, and up to 20 diagnoses from each hospital contact. Diagnoses have since 1994 been classified according to the International Classification of Diseases (ICD) 10th edition. The ICD-10 codes used to identify patients with liver metastases was C78.7 and additional codes used for identification of the primary cancers were C18-21 (CRC/anal cancer) and C00-17; and
C22-97 (non-CRC). ${ }^{12}$ We restricted our study population to patients considered to have synchronous metastases, defined by being registered with a code for a primary cancer at date of liver metastasis diagnosis or within 60 days thereafter.

\section{Survival}

Survival status and eventual date of death for all included patients was obtained through the Civil Registration System. Since 1968, this system has kept electronic records, updated daily, on date of birth, date of emigration, and vital status for all residents. Data linkage was performed using the civil registration number as identification number.

\section{Statistical analysis}

Because we defined our population as patients with a primary cancer diagnosed at date of liver metastasis diagnosis or up to 60 days thereafter, we started our follow-up 60 days after metastasis diagnosis. We followed each patient until emigration, death, or 25 June 2010, whichever came first. To visualize crude survival we constructed Kaplan-Meier curves stratified according to period of diagnosis (1998-2000, 2001-2003, 2004-2006, and 2007-2009). We estimated 1-, $3-$, and 5-year survival. In the latter periods we estimated 3- and 5-year survival using a hybrid analysis in which we included the actual survival for as long as possible and then estimated the conditional probability of surviving thereafter based on the corresponding survival experience of patients in the previous period (ie, using a period analysis technique). ${ }^{13}$ To compare mortality over time we used Cox proportional hazards regression analysis with 1998 to 2000 as the reference period to estimate the MRR and the corresponding $95 \%$ confidence interval $(\mathrm{CI})$ adjusting for age (15-59 years, 60-74 years, $75+$ years) and gender. The assumptions of proportional

Table I Patients with synchronous liver metastasis by primary tumor site, Northern Denmark 1998 to 2009

\begin{tabular}{lll}
\hline Site & Frequency & Percent \\
\hline Colorectal (CRC) & 541 & 52.99 \\
Noncolorectal (non-CRC) & 480 & 47.01 \\
Pancreas & 144 & 14.10 \\
Lung, bronchi, and trachea & 130 & 12.73 \\
Stomach & 38 & 3.72 \\
Liver & 27 & 2.64 \\
Breast & 22 & 2.15 \\
Esophagus & 21 & 2.06 \\
Kidney & 19 & 1.86 \\
Gallbladder and biliary tract & 16 & 1.57 \\
Other & 63 & 6.17 \\
Total & 1021 & 100.00 \\
\hline
\end{tabular}

Abbreviation: CRC, colorectal carcinoma. 

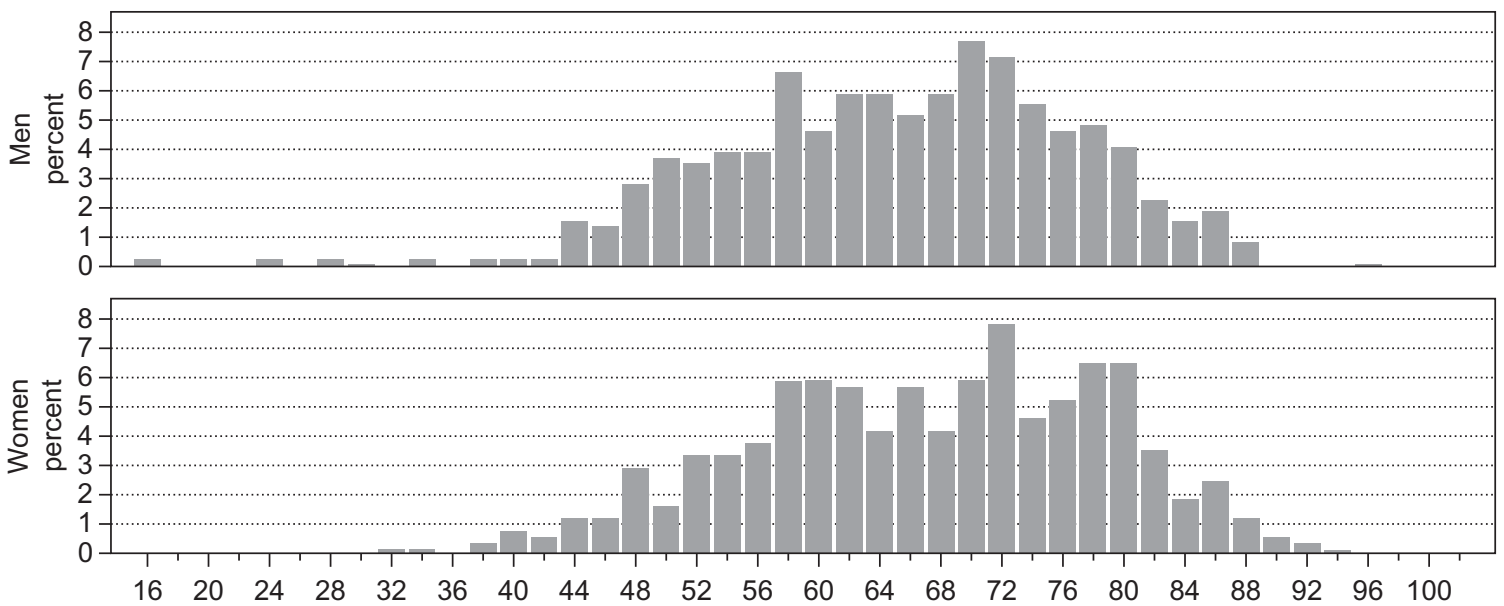

Figure I Age distribution of the total cohort of 102 I patients diagnosed with liver metastases, Denmark I998 to 2009.

hazards were examined graphically by plotting observed and simulated paths of the standardized score process.

Analyses were performed using SAS version 9.2 (SAS Institute Inc., Cary, NC).

\section{Results}

The study cohort comprised a total number of 1021 patients identified with synchronous liver metastases during the 12 year period 1998 to 2009 in the Central and North Denmark Regions who all survived at least 60 days after date of diagnosis. The largest group of patients $(\mathrm{n}=541 ; 53.0 \%)$ were diagnosed with CRC as primary cancer whereas the remaining non-CRC patients represented a variety of tumor sites $(n=480 ; 47.0 \%)$. In the same time period 15,097 patients were registered with primary CRC (colon cancer or rectal cancer; ICD: C18-19 or C20-21) in the Central and North Denmark Regions. The distribution of patients according to the primary tumor site is listed in Table 1 . The non-CRC group was dominated by patients with primary gastro-intestinal and lung cancer, whereas only $2.2 \%$ had a primary breast cancer. In the total cohort the median age was 66.8 (range 15.3-95.3) years (Figure 1) with a male/female distribution of $54 \% / 46 \%$. The median age was 67.7 (range 27.0-93.2) and 66.2 (range 15.3-95.3) years and the male/ female distribution 53\%/47\% and 55\%/45\% for the CRC and non-CRC cohorts, respectively.

For patients with synchronous liver metastases, the overall survival improved over the 12-year period (Table 2). This was most pronounced during 2007 to 2009. Over the time period 1998 to 2009 , the 5-year survival improved from 3\% (95\% CI: $1 \%-6 \%)$ to $10 \%(95 \% \mathrm{CI}: 6 \%-14 \%)$ (predicted value). The 5 -year survival of CRC-patients

Table 2 I-, 3-, and 5-year overall survival and mortality rate ratio (MRR) adjusted for age and gender for Danish patients with liver metastases stratified by period of diagnosis

\begin{tabular}{|c|c|c|c|c|}
\hline & \multicolumn{4}{|c|}{ Period of diagnosis } \\
\hline & $1998-2000$ & $200 I-2003$ & 2004-2006 & 2007-2009 \\
\hline Number of cancer patients & 190 & 288 & 239 & 304 \\
\hline Median age (years) & 67 & 67 & 66 & 68 \\
\hline \multicolumn{5}{|l|}{ I-year } \\
\hline Survival & $22 \%(16 \%-28 \%)$ & $25 \%(20 \%-30 \%)$ & $24 \%(19 \%-30 \%)$ & $30 \%(25 \%-36 \%)$ \\
\hline MRR & I (reference) & $0.96(0.78-1.18)$ & $0.88(0.7 I-1.09)$ & $0.76(0.62-0.94)$ \\
\hline Adjusted MRR* & I (reference) & $0.98(0.79-1.20)$ & $0.87(0.70-1.08)$ & $0.74(0.60-0.92)$ \\
\hline \multicolumn{5}{|l|}{ 3-year } \\
\hline Survival & $5 \%(3 \%-9 \%)$ & $7 \%(5 \%-11 \%)$ & $5 \%(3 \%-9 \%)$ & $12 \%(8 \%-16 \%) \dagger$ \\
\hline MRR & I (reference) & $0.94(0.78-1.13)$ & $0.91(0.75-1.10)$ & $0.77(0.63-0.93) \dagger$ \\
\hline Adjusted MRR* & I (reference) & $0.96(0.79-1.16)$ & $0.91(0.75-1.11)$ & $0.75(0.62-0.91) \dagger$ \\
\hline \multicolumn{5}{|l|}{ 5-year } \\
\hline Survival & $3 \%(1 \%-6 \%)$ & $5 \%(3 \%-7 \%)$ & $4 \%(2 \%-7 \%) \dagger$ & $10 \%(6 \%-14 \%) \dagger$ \\
\hline MRR & I (reference) & $0.93(0.77-1.13)$ & $0.90(0.74-1.09) \dagger$ & $0.76(0.63-0.92) \dagger$ \\
\hline Adjusted MRR* & I (reference) & $0.95(0.79-1.15)$ & $0.90(0.74-1.10) \dagger$ & $0.75(0.62-0.90) \dagger$ \\
\hline
\end{tabular}

Notes: *Adjusted for age and gender; †Predicted values. 
Table 3 I-, 3-, and 5-year overall survival and mortality rate ratio (MRR) adjusted for age and gender for the cohort of colorectal carcinoma liver metastasis patients stratified by period of diagnosis

\begin{tabular}{|c|c|c|c|c|}
\hline & \multicolumn{4}{|c|}{ Year of diagnosis } \\
\hline & $1998-2000$ & $200 I-2003$ & 2004-2006 & 2007-2009 \\
\hline Number of cancer patients & 103 & 158 & 120 & 160 \\
\hline Median age (years) & 67 & 67 & 66 & 69 \\
\hline \multicolumn{5}{|l|}{ I-year } \\
\hline Survival & $28 \%(20 \%-37 \%)$ & $37 \%(30 \%-45 \%)$ & $36 \%(27 \%-44 \%)$ & $46 \%(37 \%-53 \%)$ \\
\hline MRR & I (reference) & $0.85(0.63-1.15)$ & $0.86(0.62-1.18)$ & $0.65(0.48-0.89)$ \\
\hline Adjusted MRR* & I (reference) & $0.90(0.67-1.22)$ & $0.85(0.61-1.17)$ & $0.62(0.45-0.85)$ \\
\hline \multicolumn{5}{|l|}{ 3-year } \\
\hline Survival & $5 \%(2 \%-10 \%)$ & $11 \%(7 \%-17 \%)$ & $9 \%(5 \%-15 \%)$ & $15 \%(9 \%-21 \%) \dagger$ \\
\hline MRR & I (reference) & $0.82(0.63-1.06)$ & $0.86(0.66-1.13)$ & $0.69(0.53-0.90) \dagger$ \\
\hline Adjusted MRR* & I (reference) & $0.87(0.67-1.12)$ & $0.87(0.66-1.15)$ & $0.68(0.52-0.88) \dagger$ \\
\hline \multicolumn{5}{|l|}{ 5-year } \\
\hline Survival & $1 \%(0 \%-5 \%)$ & $6 \%(3 \%-11 \%)$ & $6 \%(3 \%-12 \%) \dagger$ & $11 \%(6 \%-18 \%) \dagger$ \\
\hline MRR & I (reference) & $0.79(0.62-1.03)$ & $0.84(0.64-1.09) \dagger$ & $0.68(0.52-0.88) \dagger$ \\
\hline Adjusted MRR* & I (reference) & $0.84(0.65-1.08)$ & $0.87(0.66-1.14) \dagger$ & $0.66(0.5 \mathrm{I}-0.86) \dagger$ \\
\hline
\end{tabular}

Notes: *Adjusted for age and gender; †Predicted values.

improved from 1\% (95\% CI: $0 \%-5 \%)$ to $11 \%(95 \% \mathrm{CI}$ : $6 \%-18 \%$ ) (Table 3) whereas the increase of survival for non-CRC patients was 5\% (95\% CI: $1 \%-10 \%)$ to $8 \%(95 \%$ CI: 4\%-14\%) (Table 4).

MRRs adjusted for age and gender in 2007 to 2009 were 0.74 (95\% CI: 0.60-0.92), 0.75 (95\% CI: 0.62-0.91) and 0.75 (95\% CI: 0.62-0.90) after 1, 3, and 5 years, respectively, compared with 1998-2000 (Figure 2A, Table 2). For CRC patients (Figure 2B) the age and gender adjusted MRRs were 0.62 (95\% CI: 0.45-0.85), 0.68 (95\% CI: 0.52-0.88), and 0.66 (95\% CI: $0.51-0.86)$ after 1,3 , and 5 years, respectively, over the same time period (Table 3). For non-CRC patients the adjusted MRRs were 0.83 (95\% CI: 0.62-1.11),
0.82 (95\% CI: 0.62-1.08), and 0.81 (95\% CI: 0.62-1.07), respectively (Table 4$)$.

\section{Discussion}

In this population-based study from Northern Denmark we found that the prognosis in patients with liver metastases diagnosed synchronously with CRC or other solid tumors improved from 1998 to 2009. Overall, 1-, 3- and 5-year mortality decreased approximately $25 \%$ for the total group and among CRC patients the mortality decreased nearly $35 \%$.

Different factors affected the interpretation of our results. We conducted a population-based study in a uniform health care system with a well-defined catchment area and had

Table 4 I-, 3-, and 5-year overall survival and mortality rate ratio (MRR) adjusted for age and gender for the cohort of non-colorectal carcinoma liver metastasis patients stratified by period of diagnosis

\begin{tabular}{|c|c|c|c|c|}
\hline & \multicolumn{4}{|c|}{ Year of diagnosis } \\
\hline & $1998-2000$ & $200 I-2003$ & 2004-2006 & 2007-2009 \\
\hline Number of cancer patients & 87 & 130 & 119 & 144 \\
\hline Median age (years) & 65 & 65 & 65 & 67 \\
\hline \multicolumn{5}{|l|}{ I-year } \\
\hline Survival & $14 \%(8 \%-22 \%)$ & $10 \%(6 \%-16 \%)$ & $13 \%(7 \%-19 \%)$ & $14 \%(8 \%-20 \%)$ \\
\hline MRR & I (reference) & I.II (0.83-I.48) & $0.81(0.60-1.09)$ & $0.83(0.62-1.11)$ \\
\hline Adjusted MRR* & I (reference) & I.II (0.83-I.48) & $0.81(0.60-1.09)$ & $0.83(0.62-1.11)$ \\
\hline \multicolumn{5}{|l|}{ 3-year } \\
\hline Survival & $6 \%(2 \%-12 \%)$ & $2 \%(1 \%-6 \%)$ & $2 \%(0 \%-5 \%)$ & $8 \%(4 \%-14 \%) \dagger$ \\
\hline MRR & I (reference) & $1.13(0.86-1.50)$ & $0.86(0.65-1.15)$ & $0.82(0.62-1.08) \dagger$ \\
\hline Adjusted MRR* & I (reference) & $1.13(0.86-1.50)$ & $0.87(0.65-1.15)$ & $0.82(0.62-1.08) \dagger$ \\
\hline \multicolumn{5}{|l|}{ 5-year } \\
\hline Survival & $5 \%(1 \%-10 \%)$ & $2 \%(1 \%-6 \%)$ & $2 \%(. \%-. \%) \dagger$ & $8 \%(4 \%-14 \%) \dagger$ \\
\hline MRR & I (reference) & I.II (0.84-I.46) & $0.86(0.65-1.14) \dagger$ & $0.82(0.62-1.08) \dagger$ \\
\hline Adjusted MRR* & I (reference) & I.II (0.84-I.47) & $0.83(0.62-1.10) \dagger$ & $0.8 \mathrm{I}(0.62-1.07) \dagger$ \\
\hline
\end{tabular}

Notes: *Adjusted for age and gender; †Predicted values. 
A

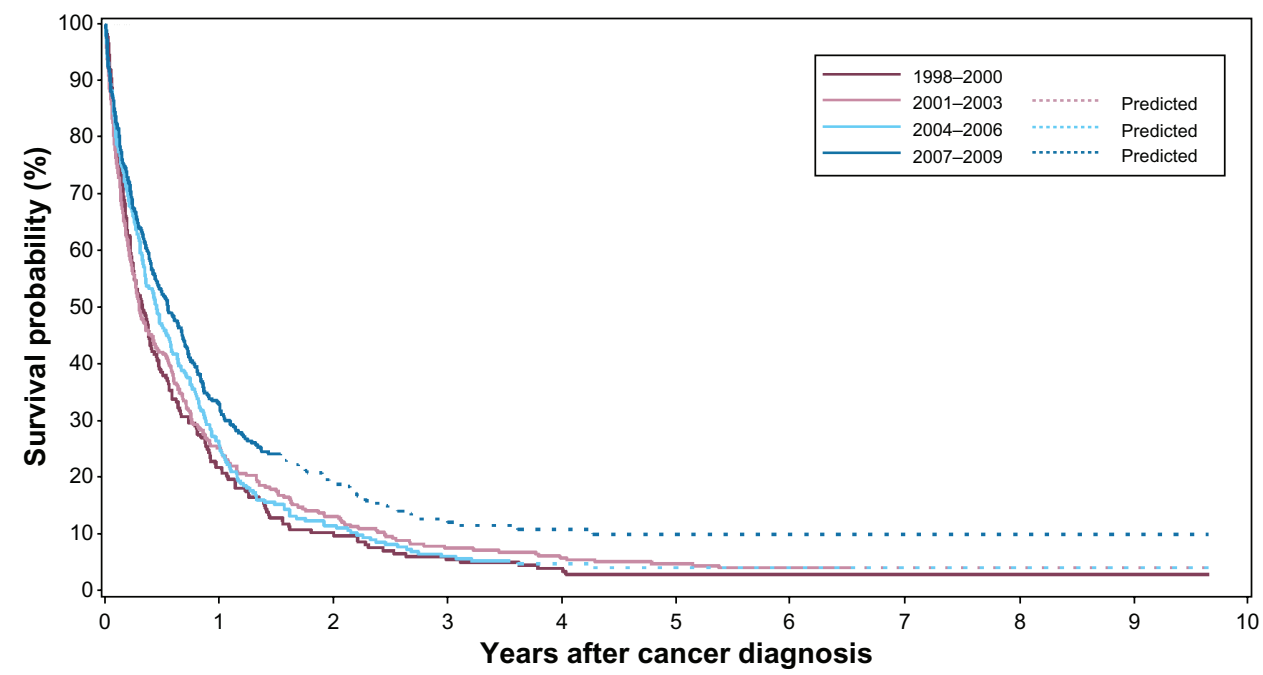

B

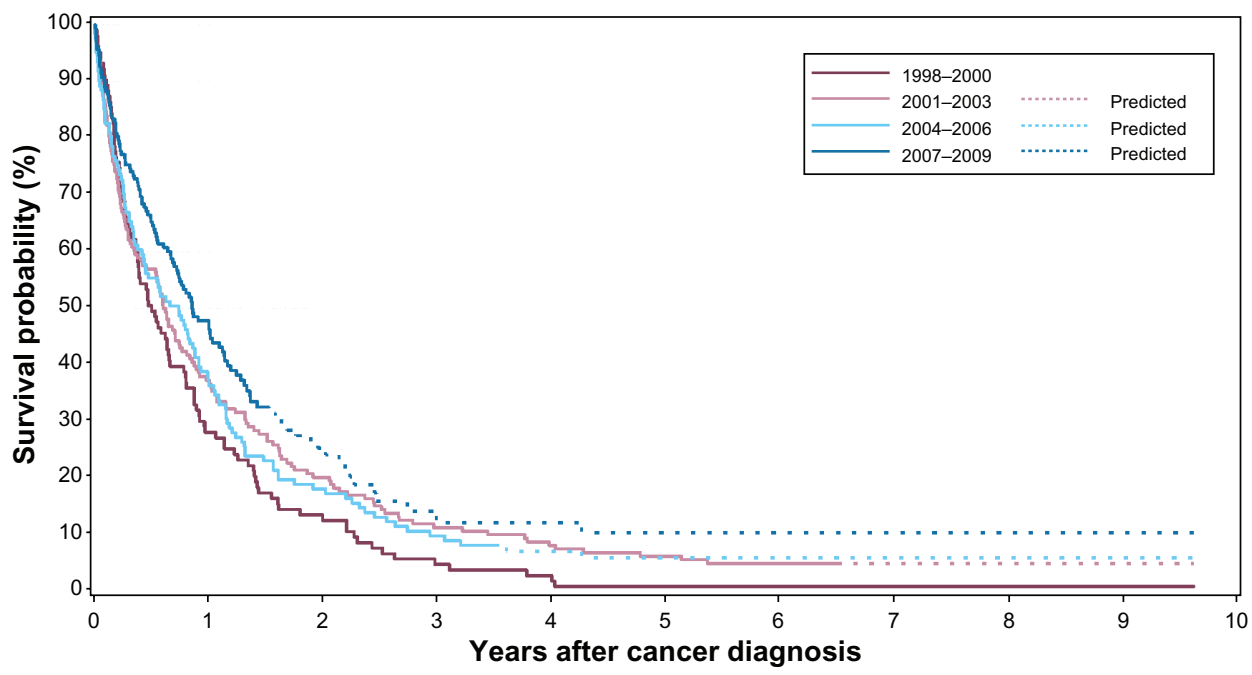

C

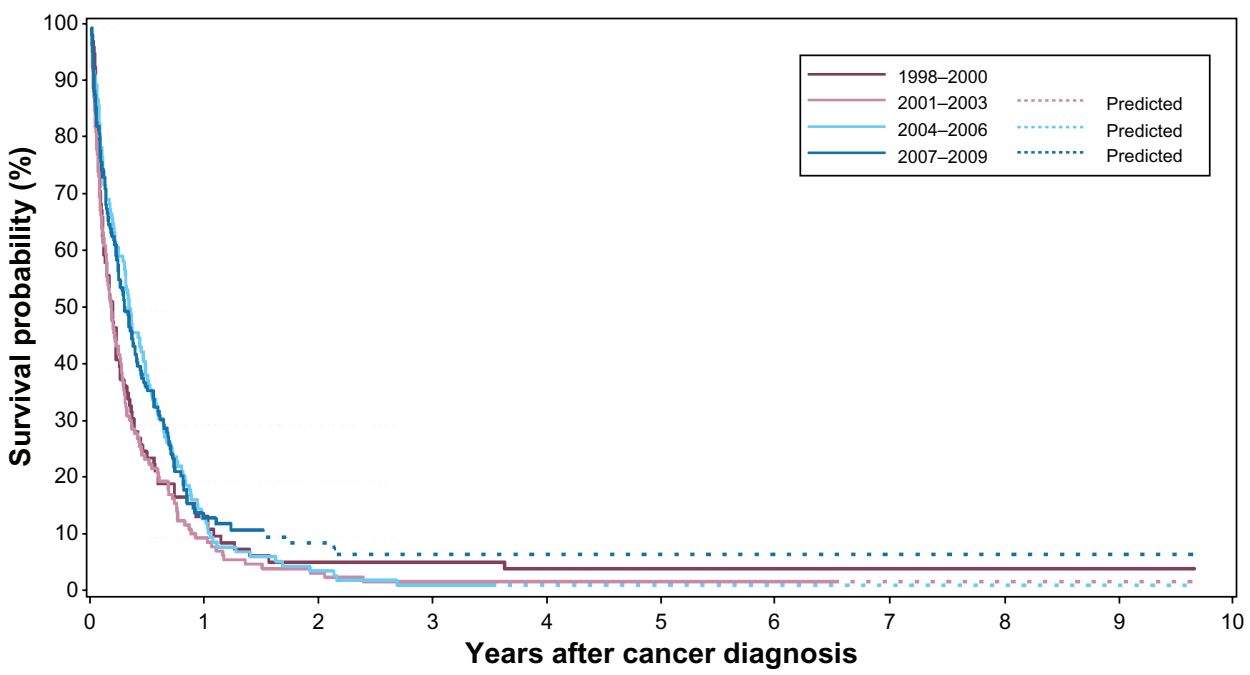

Figure 2 Crude survival of $\mathbf{A})$ the total patient cohort with synchronous liver metastases $(\mathrm{n}=102 \mathrm{I})$, B) the cohort of colorectal cancer $(C R C)$-patients with synchronous liver metastases $(n=541)$, and $C)$ the cohort of non-CRC patients with synchronous liver metastases $(n=480)$, stratified by period of diagnosis. 
data on a population of patients treated over more than a decade. Moreover, we had virtually complete follow-up for mortality. However, our study also had limitations. The registration of liver metastases in DNRP may not be complete and may have changed during the study period. Since completeness of liver metastases in DNPR may differ by disease severity our survival estimates could be inaccurate. Nevertheless, the annual number of CRC patients with synchronous liver metastases did not increase during our study period which speaks against major differences in completeness with time. Therefore, we do not expect differences in completeness of registration to explain the improved survival in CRC patients. Among non-CRC patients the annual number of patients increased. If this was caused by inclusion of more patients with less advanced liver metastases diagnosed earlier the observed improvement in survival among non-CRC patients could be explained by lead time bias. Furthermore, we only included patients with liver metastases at time of cancer diagnosis and did not include patients with liver metastases diagnosed after resection of the primary tumor (ie, metachronous metastases) so we are unable to evaluate the effect in these patients. In order to provide more up-to-date information on long-term survival we used a hybrid analysis rather than a traditional analysis. Traditionally, the 5-year survival would be derived only from patients who had been observed for 5 years or more, thereby not reflecting more recent improvements in survival. The weakness of the hybrid analysis is that it may not be as accurate as directly observed survival. However, since we based our predictions on the survival experience in the previous period of our study we expect these predictions to be conservative estimates of the improved survival.

Imaging technology used for diagnosis of liver metastases has improved during the period. Computer tomography and magnetic resonance imaging which are considered the best imaging modalities in diagnosis and staging of liver metastases have improved over time, ${ }^{14}$ and for ultrasound utilization of contrast enhancement has increased the diagnostic accuracy. ${ }^{15}$ However, since the numbers of new cases did not change for CRC, we find it unlikely that improvement of imaging technology is responsible for the entire increased number of non-CRC patients with liver metastases as noticed in the present study. In this regards, we find it most likely that the study population represents a steady-state population and that the survival benefit is caused by improved therapy.

An improvement in survival is likely to be a result of the many treatments that are offered to the patients over the entire course of the disease. During the time period of the present study a multidisciplinary team was introduced for treatment of liver tumors and the therapy for liver tumors was evolving over the period. Historical studies have shown that long-term survival can be achieved in patients undergoing surgical resection of oligometastases to the liver. ${ }^{3-5}$ Patients who are technically operable should therefore be offered a surgical resection. Novel surgical techniques have increased the number of patients who are technically operable. ${ }^{16,17}$ However, only approximately $25 \%$ of patients with CRC liver metastases are amenable to surgical resection. ${ }^{18}$ Nonsurgical ablation techniques, such as radiofrequency ablation or stereotactic body radiation therapy are frequently used to treat patients with unresectable liver metastases in Denmark. ${ }^{19,20}$ Still, the efficacy of surgery and nonsurgical tumor ablation has never been proven in randomized trials.

Traditionally, metastatic cancer is treated by systemic therapies and chemotherapy has improved considerably during the last decade. For CRC patients, chemotherapy and targeted therapy to patients with metastatic cancer may improve survival, lessen symptoms related to the disease, improve quality of life and downsize liver-only metastases in patients with nonresectable metastases that potentially may become resectable. ${ }^{21}$ And for patients with metastatic non$\mathrm{CRC}$, new cytostatic agents, hormonal agents, and biological therapies have resulted in survival benefit. ${ }^{22}$

In conclusion our study indicates an improvement in survival for liver metastases patients during the 12 years from 1998 to 2009. The survival gain for CRC liver metastasis patients may be a result of intensified efforts in the multidisciplinary care of patients who benefit from improved systemic therapy and more aggressive approach in resection or ablation of the liver metastases.

\section{Acknowledgments}

The study received financial support from the Karen Elise Jensen Foundation, Department of Clinical Epidemiology's Research Foundation and the Regional Clinical Epidemiological Monitoring Initiative for Central and North Denmark Regions.

\section{Disclosure}

Morten Høyer is supported by grants from AP Møller and hustru Chastine McKinney Møllers Fond for Almene Formål og the Lundbeck Foundation Centre for Interventional Research in Radiation Oncology (CIRRO).

\section{References}

1. Hess KR, Varadhachary GR, Taylor SH, et al. Metastatic patterns in adenocarcinoma. Cancer. 2006;106(7):1624-1633. 
2. Manfredi S, Lepage C, Hatem C, Coatmeur O, Faivre J, Bouvier AM. Epidemiology and management of liver metastases from colorectal cancer. Ann Surg. 2006;244(2):254-259.

3. Fong Y, Fortner J, Sun RL, Brennan MF, Blumgart LH. Clinical score for predicting recurrence after hepatic resection for metastatic colorectal cancer: analysis of 1001 consecutive cases. Ann Surg. 1999;230(3): 309-318.

4. House MG, Ito $\mathrm{H}$, Gonen $\mathrm{M}$, et al. Survival after hepatic resection for metastatic colorectal cancer: trends in outcomes for 1,600 patients during two decades at a single institution. J Am Coll Surg. 2010;210(5): 744-745.

5. Nordlinger B, Guiguet M, Vaillant JC, et al. Surgical resection of colorectal carcinoma metastases to the liver. A prognostic scoring system to improve case selection, based on 1568 patients. Association Francaise de Chirurgie. Cancer. 1996;77(7):1254-1262.

6. Smith MD, McCall JL. Systematic review of tumour number and outcome after radical treatment of colorectal liver metastases. Br J Surg. 2009;96(10):1101-1113.

7. Wong SL, Mangu PB, Choti MA, et al. American Society of Clinical Oncology 2009 clinical evidence review on radiofrequency ablation of hepatic metastases from colorectal cancer. J Clin Oncol. 2010;28(3):493-508.

8. Cunningham D, Atkin W, Lenz HJ, et al. Colorectal cancer. Lancet. 2010;375(9719):1030-1047.

9. Sundhedsstyrelsen. Cancer Treatment Plans in Denmark. http://www. sst.dk/. 2011.

10. Pedersen CB, Gotzsche H, Moller JO, Mortensen PB. The Danish Civil Registration System: a cohort of eight million persons. Dan Med Bull. 2006;53(4):441-449.

11. Andersen TF, Madsen M, Jorgensen J, Mellemkjoer L, Olsen JH. The Danish National Hospital Register: a valuable source of data for modern health sciences. Dan Med Bull. 1999;46(3):263-268.
12. WHO. International Statistical Classification of Diseases and Related Health Problems, 10th Revision. http://apps.who.int/classifications/ apps/icd/icd10online/. 2011.

13. Brenner H, Rachet B. Hybrid analysis for up-to-date long-term survival rates in cancer registries with delayed recording of incident cases. Eur J Cancer. 2004;40(16):2494-2501.

14. Floriani I, Torri V, Rulli E, et al. Performance of imaging modalities in diagnosis of liver metastases from colorectal cancer: a systematic review and meta-analysis. J Magn Reson Imaging. 2010;31(1):19-31.

15. Larsen LP. Role of contrast enhanced ultrasonography in the assessment of hepatic metastases: A review. World J Hepatol. 2010;2(1):8-15.

16. Chun YS, Vauthey JN. Extending the frontiers of resectability in advanced colorectal cancer. Eur J Surg Oncol. 2007;33(Suppl 2):S52-S58.

17. Khatri VP, Petrelli NJ, Belghiti J. Extending the frontiers of surgical therapy for hepatic colorectal metastases: is there a limit? J Clin Oncol. 2005;23(33):8490-8499.

18. Scheele J, Stang R, Tendorf-Hofmann A, Paul M. Resection of colorectal liver metastases. World J Surg. 1995;19(1):59-71.

19. Sorensen SM, Mortensen FV, Nielsen DT. Radiofrequency ablation of colorectal liver metastases: long-term survival. Acta Radiol. 2007;48(3): 253-258.

20. Hoyer M, Roed H, Traberg HA, et al. Phase II study on stereotactic body radiotherapy of colorectal metastases. Acta Oncol. 2006;45(7): 823-830.

21. Pagani O, Senkus E, Wood W, et al. International guidelines for management of metastatic breast cancer: can metastatic breast cancer be cured? J Natl Cancer Inst. 2010;102(7):456-463.

22. D'Addario G, Fruh M, Reck M, Baumann P, Klepetko W, Felip E. Metastatic non-small-cell lung cancer: ESMO Clinical Practice Guidelines for diagnosis, treatment and follow-up. Ann Oncol. 2010;21(Suppl 5): v116-v119.
Clinical Epidemiology

\section{Publish your work in this journal}

Clinical Epidemiology is an international, peer-reviewed, open access journal focusing on disease and drug epidemiology, identification of risk factors and screening procedures to develop optimal preventative initiatives and programs. Specific topics include: diagnosis, prognosis, treatment, screening, prevention, risk factor modification, systematic

\section{Dovepress}

reviews, risk \& safety of medical interventions, epidemiology \& biostatical methods, evaluation of guidelines, translational medicine, health policies \& economic evaluations. The manuscript management system is completely online and includes a very quick and fair peer-review system, which is all easy to use. 